To appear in H. De Preester \& M. Tsakiris (2018). The Interoceptive Basis of Mind. Oxford University Press. Penultimate version.

\title{
From physiology to experience: enriching existing conceptions of "arousal" in affective science
}

\author{
Giovanna Colombetti \\ Department of Sociology, Philosophy and Anthropology \\ University of Exeter \\ \& \\ Neil Harrison \\ Department of Neuroscience, Brighton \& Sussex Medical School, \\ University of Sussex
}

\begin{abstract}
We examine the notion of "arousal", an influential notion in affective science referring to the degree of an individual's "activation" or "excitement" during an emotional state. We examine this notion specifically in relation to interoception, defined broadly as "sensitivity to stimuli arising inside the organism". We first distinguish "physiological arousal" from "experienced arousal" and argue that both need to be characterised more broadly than commonly done. Physiological arousal does not reduce to sympathetic activation, as it involves complex interactions between multiple functionally distinct pathways within sympathetic and parasympathetic divisions of the autonomic nervous system, as well as endocrine and immune systems, and even the gut microbiota. Relatedly, experienced arousal does not reduce to the perception of changes in the body sensed by visceral afferents in response to autonomic nervous system activity, but also includes humorally mediated interoceptive pathways, somatic sensations of various kinds, and "background" bodily feelings.
\end{abstract}

Keywords: arousal; emotion; interoception; autonomic nervous system; emotional experience; psychoneuroendocrinology; psychoneuroimmunology; microbiome. 


\section{Introduction}

"Arousal" is a key notion in the interdisciplinary field of "affective science", which includes primarily the psychology and neuroscience of emotion, but also philosophical and computational approaches to emotion (Davidson et al., 2003, Scarantino, forthcoming). Roughly and preliminarily, we can say that "arousal" refers to how more or less "excited" or "activated" one is during an emotion; for example, someone who is very scared is often said to be highly aroused, whereas sadness and contentment are often regarded as involving low degrees of arousal. Several affective scientists regard arousal not just as an important dimension of emotion, but even as a necessary one: part of what it is to be in an emotional state is to be more or less aroused (e.g., Russell, 2003). Importantly for the topic of this volume, arousal is often regarded as interlinked with interoception.

But what is it to be aroused during an emotion, exactly? As it turns out, no short definition can capture the various meanings that the term "arousal" has in affective science; moreover, these different meanings are often not clearly discriminated (Colombetti \& Kuppens, forthcoming). One goal of this chapter is to bring some clarity by distinguishing the two main meanings of this term, i.e., what we call physiological and experienced arousal. ${ }^{1}$ Another goal is to clarify the relationship between these two meanings of arousal, and interoception, understood broadly as "sensitivity to stimuli arising inside the organism" - where "sensitivity" does not necessarily entail conscious perception. In particular, we argue that it is restrictive and inaccurate to reduce physiological arousal to a single dimension of sympathetic activation, or even to just autonomic activation ${ }^{2}$ (psychological studies that include

${ }^{1}$ In this chapter we limit our analysis to arousal as a component or dimension of emotion and other affective states, such as moods.

2 "Autonomic activation" commonly refers to activation of the autonomic nervous system (ANS). The ANS is a division of the peripheral nervous system, and is itself divided into sympathetic and parasympathetic nervous system. The other two divisions of the peripheral nervous system are the somatic nervous system, which controls the voluntary muscles and more generally the musculoskeletal system, and the enteric nervous system, which both alone and together with the ANS controls 
"physiological measurements", for example, often measure only a few dimensions of autonomic activation, such as skin conductance responses, heart rate and blood pressure; see Fox, 2008, pp. 32-34). As for experienced arousal, we argue, relatedly, that it is restrictive and inaccurate to reduce it to the conscious perception of organismic changes signalled just via visceral afferents (the afferent partner of the ANS). Experienced arousal, we suggest, additionally includes the perception of circulating substances mediated via humoral interoceptive pathways, as well as various somatic sensations and what we call "background bodily feelings".

\section{Two main meanings of "arousal"}

In contemporary affective science, "arousal" has two main meanings. First, it refers to what we term experienced arousal, i.e., the lived, first-personal or subjective experience of being (more or less) aroused during an emotion. Second, it refers to what we term physiological arousal, i.e., the third-personal or objective biological processes that occur in the organism during an emotion. These two meanings of arousal are clearly different, but they are sometimes conflated, and it is often assumed that experienced arousal provides veridical information about physiological arousal. For example, Scherer \& Wallbott (1994) addressed the question of whether at least some emotions exhibit the same patterns of "physiological symptoms" (their term) across cultures. To do this, they used questionnaires that asked participants how they felt their body when experiencing various emotions, but did not actually record any physiological measurements. Likewise, Grewe et al. (2007) studied "physiological responses" to music with questionnaires asking participants to "report their perceived bodily reactions" (p. 779). They actually also measured physiological responses to music in the form of skin conductance and facial muscle activity. Throughout the paper, however, they conflate "physiological changes" with "reported (or experienced) physiological changes". More recently, Nummenmaa et al. (2014) identified different "bodily sensations maps" for 13 different emotions. Although they make it clear that this study was about experienced arousal, they assume throughout their paper that bodily sensations "represent" (their term) physiological processes.

activity within the gastrointestinal tract. Until recently the enteric nervous system was regarded as a part of the ANS; it is now recognised as a mainly independent system. 
How experienced arousal relates to physiological arousal, however, needs to be assessed empirically. Suppose you are feeling very agitated (experienced arousal) as part of being worried about an imminent job interview. Your feeling of agitation may include specific sensations such as feeling your heart pounding heavily in your chest, and your mouth and throat being dry. Now, it is natural and not implausible in this case to think that your heart is in fact beating differently from when you are calmer, and that your mouth and throat are in fact drier (physiological arousal). Whether this is really the case, however, needs to be confirmed by conducting actual measurements on the state of your heart, mouth and throat. One cannot simply infer the physiological condition of any specific body part from how the person feels, for a variety of reasons. One is that the person's reports of her bodily feelings may be influenced by "social schemata" (Rimé et al., 1990), i.e., learnt templates of how one is expected to feel in specific situations. Another reason is that there are individual differences in how accurately people can perceive their actual bodily changes (usually measured with heartbeat detection tasks) - a capacity termed "interoceptive accuracy" (Garfinkel et al., 2015). Furthermore, the same person can be more or less accurate depending on the task performed, context, stress, etc. (Schulz et al., 2013). Finally, even though some of us can, at times, accurately feel what is going on in some parts of our body, there is much going on in our body that is consciously inaccessible (Critchley \& Harrison, 2013). For example, we cannot feel our pupils dilating or our blood pressure rising. Thus, how bodily aroused a person feels (experienced arousal) provides at best only a partial look into her physiological arousal. At worst, it provides an inaccurate or distorted view of the latter.

The upshot is that we need to distinguish clearly between the subjective experience, or feeling, of being aroused, excited or activated (all terms found in the literature and used as synonyms), and what is actually going on in the organism during an emotional episode. Having clarified this, let us now examine both phenomena more closely.

\section{Physiological arousal}


That our organism often undergoes physiological changes during emotional episodes is something we can easily witness, and often do: we see our hands shaking when we are nervous, and a mirror can show our skin getting red when we are embarrassed. This is such a commonplace observation that it is not surprising to find it in ancient philosophy texts. At the beginning of De Anima, for example, Aristotle noted that in "anger, mildness, fear, pity, hope and even joy and love and hating ... the body is affected in some way" (1986, p. 128). The Stoics and Galen also recognised the contribution of the body to our emotional states (Gill, 2010).

There is thus a sense in which we have always known that our body gets more or less "excited" during different emotions. It is worth noting, though, that contemporary empirical studies of emotion typically measure physiological arousal by measuring something very specific, i.e., changes in the organism ascribed (sometimes incorrectly) to activation of the sympathetic division of the ANS, such as increases in heart rate and skin conductance, alterations in skin temperature, and pupillary dilation (Berntson \& Cacioppo, 2009). ${ }^{3}$ Walter Cannon famously associated the sympathetic nervous system with the "fight or flight response", and the narrow identification of physiological arousal with sympathetic activation can be traced back to work on fear and rage he conducted in the 1910s and 1920s (Cannon, 1929). Cannon also conceptualised sympathetic activation as mutually exclusive with, and antagonistic to, activation of the parasympathetic system, whose contribution to emotion he generally disregarded. Finally, Cannon is also responsible for characterising sympathetic activation as generally uniform and undifferentiated. Though these ideas were challenged by Cannon's contemporaries (see Dror, 2014), they influenced scientific conceptions of arousal throughout the $20^{\text {th }}$ century, and still do so. Most famously, Schachter \& Singer (1962, pp. 381-382) maintained that the same state of (sympathetic) physiological arousal (induced using an adrenaline injection) "could be labelled 'joy' or 'fury' or 'jealousy' or any of a great diversity of emotional labels depending on the cognitive aspects of the situation". These authors also suggested (despite previous studies to the contrary, e.g., Ax, 1953; Wolf \& Wolff, 1947), that "emotional states may ... be generally characterized by a high

\footnotetext{
${ }^{3}$ Acute increases in heart rate and pupil size are actually initiated by a withdrawal in parasympathetic tone (Robinson et al., 1966; Barbur, 2004).
} 
level of sympathetic activation with few if any physiological distinguishers among the many emotional states" (Schachter \& Singer, 1962, pp. 397).

Our view is that this conception of arousal during emotion is superseded and too narrow, and needs to be abandoned, for the following four reasons.

(1) The term "autonomic nervous system" and its division into sympathetic and para(meaning "by the side of", "alongside") sympathetic components was introduced by Langley (1900) on the basis of predominantly neuroanatomical, rather than functional, considerations. To talk of global sympathetic and parasympathetic functions (e.g., fight-and-flight vs. rest-and-digest) has the potential to generate misunderstandings and to create an overly simplistic impression of the functional architecture of the ANS (for more details, see Harrison et al., 2013). For example, empirical data acquired over the last half-century show that pre- and postganglionic neurons of both the sympathetic and parasympathetic nervous system link together in multiple functionally distinct pathways that facilitate the generation of a huge variety of highly differentiated and specific responses (Jänig, 2006). This has undermined previous false assumptions that sympathetic preganglionic neurons diverge widely and synapse with postganglionic neurons with multiple diverse functions, dispelling the belief that the sympathetic nervous system operates in a monolithic all-or-nothing fashion. This research also demonstrates that the ANS can support emotion-specific physiological patterning.

(2) A consideration that invites broadening the traditional conception of physiological arousal is that autonomic activation is also influenced by afferent (from periphery to brain) neural and humoral feedback pathways (for details see Critchley \& Harrison, 2013). Visceral afferent fibres innervate almost all tissues of the body and fall into two broad groups: firstly, those that carry motivational information, e.g., hunger, satiety, thirst, nausea, and respiratory sensations, and travel mainly along cranial, e.g., vagus and glossopharyngeal, nerves to terminate within the nucleus of the solitary tract; secondly, spinal visceral afferents that project to the dorsal horns of the spinal cord and, via spinal laminar 1, into the spinothalamic tract. These fibres tend to have a more prominent role in signalling tissue damage. Humoral feedback is largely processed through the circumventricular organs (regions of the brain that lack 
a normal blood-brain barrier), though some - e.g., core temperature, glucose, and insulin - can also be sensed directly within brain regions such as the hypothalamus. Additionally, inflammatory mediators can modulate brain function through microglial transduction pathways, resulting in a wave of microglial activation that propagates across the brain (Rivest, 2009; Saper et al., 2012). Efferent activation is continuously modulated by this afferent, "interoceptive" feedback, so that it is misleading to restrict autonomic arousal occurring during emotion only to the outcome of neural efferent processes, without including the continuous regulatory afferent feedback that cooccurs with those processes.

(3) A further and partly related challenge to narrow conceptions of physiological arousal comes from psychoneuroendocrinology and psychoneuroimmunology. Developments in these fields have shown that the central and peripheral nervous system bi-directionally interact with both endocrine and immune processes, and that these interactions influence, and are influenced by, our emotional states. We know for example that the stress response includes the release of hormones from the brain into the adrenal glands and the bloodstream, which in turn influence hypothalamic-pituitary activity in the brain (Charmandari et al., 2005; Spiga et al., 2015). We also know that, during illness, the immune system produces proinflammatory proteins (cytokines) that influence brain activity (Harrison, 2017) and that appear to play a contributory role in at least some patients with depression (Dantzer et al., 2008); in turn, the brain responds by sending signals to inhibit this inflammatory process (Tracey, 2002). Given this bidirectional interactivity, in our view it is arbitrary to identify arousal with activation of any one system alone (the ANS, the endocrine system, etc.), or of any subset of it. Bidirectional interactivity also implies that it would still be arbitrary to regard the ANS (or any other system alone) as the "most relevant" or "most basic" arousal system, with the other systems making only a "peripheral contribution" to arousal. ${ }^{4}$ The existence of reciprocal influences entails that the systems involved are coupled, such that, without additional criteria or reasons, no system alone can be picked out as the one having the causally most relevant role. In the presence of this complexity, we think it more plausible to regard the combined activity of all systems involved as constituting physiological arousal.

\footnotetext{
${ }^{4}$ Thanks to an anonymous reviewer for suggesting this option.
} 
The reason why endocrine and immune changes are generally not included in definitions of physiological arousal may have to do with the widespread assumption, in affective science, that emotions are short-lived episodes that involve brief but intense changes in the body. Changes in the endocrine and immune systems are typically regarded to unfold on a longer timeframe, and thus arguably do not qualify as candidates for arousal. Indeed, sometimes arousal in emotion is explicitly characterised as "phasic" (temporary, short-lived; see, e.g., Fowles, 2009, p. 50), which excludes longer-lasting physiological processes (see also Bradley \& Lang, 2007 , p. 601). But it is not obvious that emotions are always short lived: whereas sometimes we are upset, annoyed or scared for a few seconds or minutes, we are also often upset, annoyed or scared (as well as jealous, envious, angry, happy, and so on) for hours or even longer. Arousal, in the latter cases, may well involve physiological processes that unfold and change over hours or even days. Moreover, it's not just emotions, defined as short-lived affective episodes, that involve a certain level of arousal; moods, often characterised as lasting longer than emotions, also do (see Thayer, 1996). Perhaps, one might suggest, the main difference between emotions and moods is precisely that, in the former, physiological arousal corresponds to brief patterns of activation of the ANS, whereas in the latter it also involves longer-lasting endocrine and immune changes. ${ }^{5}$ This is in part, of course, an empirical question. Yet, importantly, emotions typically occur in the context of a mood that makes some emotions more likely than others (e.g., one is more likely to get angry at someone when in an irritable mood); the physiological profile of a certain mood is thus likely to affect the one of these emotions, so that short-lived activation of the ANS would occur in a specific endocrine and immune context, which should then be regarded as part and parcel of the physiological arousal profile of the emotions in question.

(4) Yet another challenge to narrow conceptions of physiological arousal comes from research on the bacteria that live in our organism. They are found in almost all parts of the body, with the highest concentration in the guts. The human guts contain nearly $10^{14}-10^{15}$ bacteria, which is $10-100$ times the number of eukaryotic cells of the

\footnotetext{
${ }^{5}$ Thanks again to an anonymous reviewer for raising this possibility.
} 
human organism $\left(10^{13}\right)$. The many different functions of these bacteria have only begun to be revealed. Importantly, we now know that they influence, and are influenced by, the central nervous system, along the so-called "microbiota-gut-brain" axis. Particularly relevant for present purposes is recent evidence indicating that stress-related mood disorders, such as anxiety and depression, alter the composition of gut bacteria, and that, in turn, the composition of gut bacteria influences those states (for reviews, see Cryan \& Dinan, 2012; Foster \& McVey Neufeld, 2013; Mayer et al., 2014). In a landmark study on mice, Sudo et al. (2004) showed that gut microbiota influence the development of the hypothalamic-pituitary-adrenal system, responsible for the endocrine response to stress. Since then, further evidence has been gathered indicating that, in humans too, microbiota influence brain processes and behaviours relevant to anxiety-related stress disorders, and even individuals' susceptibility to depression (see reviews listed above for references). This influence appears to occur via neural, hormonal, and immune routes: many of the effects of gut microbiota on brain and behaviour are dependent on activation of visceral afferents travelling in the vagus nerve; gut microbiota also generate neurotransmitters and neuromodulators known to influence mood, such as GABA, serotonin, noradrenaline, dopamine and acetylcholine; and gut microbiota can also influence circulating levels of pro-inflammatory cytokines produced by innate immune cells, which, as we saw, affect brain function. Less is known about the relation between microbiota and short-lived emotions, but given the influence of moods on the latter, microbiota are likely to influence them as well.

Conceptually, this body of work raises the question of where the natural boundaries of physiological arousal lie: what is the physical entity that gets aroused? The more conservative answer is that physiological arousal recruits subsystems and processes of the organism "traditionally conceived" (i.e., formed by cells with the same DNA), and that gut microbiota are different living forms (cells with different DNA) that causally influence those subsystems and processes. In other words, bacteria are not part of the organism, and thus not of physiological arousal either; rather they constitute an external context that modulates, and is modulated by, the organism "proper". A less intuitive, yet arguably more coherent, answer is that gut microbiota can be constitutive parts of the physiological arousal that characterises affective processes. The reasoning is the same we applied earlier to the recognition of the 
existence of reciprocal influences between the sympathetic and parasympathetic system, and more generally between the central nervous system (CNS), ANS, endocrine and immune system: given the complex mutual relations interconnecting all these systems and the gut microbiota, it is arguably conceptually problematic and even arbitrary to maintain that only processes of the organism traditionally conceived can constitute physiological arousal, and that microbial processes are mere external factors or extrinsic (non-constitutive) causes. Rather, it seems more coherent to regard microbiotic processes as constitutive of physiological arousal.

In sum, together these four sets of considerations indicate that it is misleading to regard physiological arousal during an emotion as a temporary upsurge from a baseline state of "non-aroused physiology" of the organism traditionally conceived. Our physiology (i.e., the totality of the processes that contribute to sustaining our living condition) is continuously changing and shifting, with the CNS influencing, and being influenced by, a multitude of processes taking place at many different timescales in the (various divisions of) the peripheral nervous system, the endocrine and immune systems, and even beyond them.

\section{Experienced arousal}

Let us now take a closer look at the notion of experienced, or subjective, arousal. Again, we can begin by noting that there is nothing surprising or controversial in claiming that during some emotions we feel more agitated or excited than during others. Indeed, that emotions can vary in how upset or excited one feels was noted long before the birth of modern psychology. Just to mention a few examples, the Stoics distinguished the "passions" from the "good emotions", where the former are intense and overwhelming, and the latter are calm and under control (Graver, 2007). Later, and possibly under the influence of the Stoics, in his Treatise of Human Nature (1739-40 [2003]) David Hume distinguished the violent passions from the calm ones. The calm passions include the moral sentiments and the aesthetic sense, which cause "no disorder in the soul" (Treatise, 2.3.3.8) and are known more by their effects than by any immediate feeling; the violent passions (love, hate, grief, joy, pride, humility), on the other hand, are characterized by the felt quality of 
"turbulence". 6

As for contemporary affective science, Jim Russell for example characterises arousal as "one's sense of mobilization and energy" (Russell, 2003, p. 148). Similarly, Fox (2008, p. 120) writes that "arousal or activation are often interpreted as the amount of energy we feel we have available". A recent neuroscientific paper defines arousal as "the degree of activation experienced during an instance of emotion, ranging from calm to excited" (Kragel \& LaBar, 2016, p. 445).

Whereas it is relatively uncontroversial to say that we feel more or less activated during an emotion, it is surprisingly hard to specify what it is to feel more or less activated, energised, mobilised, and/or under the control of a "turbulent passion". A common view (consistent with the popular identification, discussed in the previous section, of "physiological arousal" with autonomic or even sympathetic activation) is to characterise experienced arousal as the conscious perception of bodily changes induced by the ANS. ${ }^{7}$ On this view, to feel aroused during an episode of fear, for example, is to feel one's own heart beating fast, or one's own skin sweating profusely or changing temperature (famously, this view was originally proposed by James, 1884; it is still influential today, see e.g. references at the beginning of section 2).

Intuitively, it indeed seems to be the case that these sensations contribute to feeling aroused during an emotion, and also that they contribute to the felt intensity of arousal: the more (less, respectively) one feels certain parts of one's body, the more (less) aroused one feels. By analogy, at first glance at least, this appears to be what happens when one feels sexually aroused, which partly involves feeling changes in erogenous areas of the body controlled by the ANS: the "more" one feels those

6 See Dixon (2003) for a historical overview of other philosophical accounts that distinguished the "unruly passions" from the calmer "affects" or "affections".

${ }^{7}$ In the rest of the chapter we call these, for lack of a better term, visceral sensations "of autonomic origin" or "due to the ANS", to distinguish them from other visceral sensations due to the interoception of substances circulating in the blood stream (as described in section 3). 
areas, the more sexually aroused one feels. ${ }^{8}$ Similarly, it would seem, in the case of arousal during an emotion. Moreover, the more aroused one feels, the less in control one feels; the traditionally recognised overwhelming character of a passion, in other words, appears to owe much to the uncontrollable nature of visceral sensations of autonomic origin. Finally, in addition to contributing to the intensity of experienced arousal, these sensations also appear to contribute to the intensity of an emotional experience. Back in 1964, Schachter had already proposed that experienced arousal contributes to felt emotional intensity. Relatedly, Wiens et al. (2000) showed that subjects who are better at perceiving their physiological arousal and report more subjective arousal, also experience emotions more intensely.

In our view, this is a plausible but still partial account of what it is to feel aroused during an emotion. Similarly to the case of physiological arousal discussed in the previous section, to regard experienced arousal as constituted only by sensations of bodily changes induces by the ANS is too narrow, and does not account for other ways in which we can feel aroused during an emotion; in particular: 1) some visceral sensations are due to the interoception of substances circulating in the bloodstream; 2) non-visceral somatic sensations also contribute to feeling aroused; and 3) experienced arousal also appears to involve conscious experiences that are not feelings of bodily changes. We already discussed the first point briefly in section 3 , point (2). Here we consider the other two points in turn.

1) William James (1884) already noted that emotional experience also involves the perception of bodily changes mediated by the somatic nervous system (the division of the peripheral nervous system that controls the musculoskeletal system; see note 2). We can call there "somatic sensations". Important somatic sensations that contribute to feeling aroused come from facial expressions (smiling, frowning, pouting, grinning, and so on) and from bodily posture, and also include felt urges to act in specific ways. For example, feeling angry and anxious often involves the conscious perception of one's tense facial muscles (such as tense jaws and/or forehead) and other bodily muscles (especially in the upper back, neck and

\footnotetext{
${ }^{8}$ But note that feelings of sexual arousal are likely mediated by a combination of visceral and somatic afferents.
} 
shoulders); feeling sad often involves feeling one's drooping jaws and eye corners, slouched posture, and so on.

In addition, feeling aroused during an emotion often involves the experience of wanting to move one way or the other (felt urges to act). This experience partly constitutes the motivational aspect of emotion. For example, during an aggressive face-to-face confrontation, we may experience wanting to shout at, or even physically attack, the person we are angry at. Most of the time in this kind of scenario we repress our outward behaviour because of social rules; yet even if we do not assail the opponent, the urge to do so is there, and is felt in one's own body tensing up, preparing to attack and restraining itself. Note that, especially in the moment, we may not be able to clearly discriminate these felt urges to act from other types of sensations (e.g., the angry person may also feel her heart pounding hard). Similar considerations readily apply to experiences of fear, great joy, contempt, pride, jealousy, and many others. In affective science, the term that best captures this aspect of emotion experience is action readiness awareness (Frijda, 1986, pp. 231240). "Action readiness", as the word indicates, refers to a state of being ready to act in a certain way. The awareness of this state is a bodily feeling, in the sense that it involves the conscious perception of one's own body, constituted by proprioceptive sensations of position, and state of tension or calmness. As in the case of visceral sensations of autonomic origin, felt urges to act appear to contribute significantly to experienced arousal during an emotion, and also to the intensity of the emotion: e.g., the more I want to shout at someone, the more aroused, agitated or upset I feel as part of my experience of anger, and arguably also the "more angry" or "more intensely angry" I feel.

2) The other reason why experienced arousal during emotion cannot be reduced to perception of bodily changes due to the ANS is that it arguably includes also conscious states that are not feelings of bodily changes. This possibility splits into two: (i) experienced arousal includes non-bodily experiences, i.e., experiences with no "bodily phenomenology"; (ii) experienced emotional arousal involves bodily feelings that are not feelings of the body, but feelings of the world shaped through bodily self-awareness; here, experienced emotional arousal does include bodily phenomenology, but this remains "in the background", as we explain below. 
According to (i), it is possible to be highly aroused during an emotion without feeling one's own body. Mostly philosophers supporting a cognitivist view of emotions tend to make this claim. For example, Claire Armon-Jones (1986, p. 51) writes: "whether or not I feel any twinges or palpitations, if my thoughts are totally consumed by a 'strong desire for an object which I do not possess and which belongs to another, then I can be said to feel 'extremely envious' ". On this view, one can be very worried that his child might have a life-threatening condition (for example) without experiencing any bodily sensation (neither visceral nor somatic). What constitutes feeling worried, here, is likely to be the conscious thoughts that one's child may suffer and die young (say), with the frequency and disruptive character of these thoughts determining the level of experienced arousal. Uriah Kriegel's (2015) recent account of emotional phenomenology also supports this possibility. In his view, emotional experience is reducible to a combination of cognitive, conative and algedonic (pain-pleasure) phenomenology, none of which, he argues, necessarily involves bodily feelings. He briefly suggests that conative phenomenology contributes to felt emotional intensity (p. 135), however not in virtue of any bodily sensation.

Our view is that possibility (i) is implausible. Although it is the case that "cognitive phenomenology" (thoughts, predictions, memories) can constitute much of our experience of being aroused during an emotion, it is not clear to us that it is deprived of any bodily phenomenology. We think that cognitivist accounts of this sort overlook possibility (ii), i.e., the existence of bodily feelings that are not feelings of the body, but experiences of the body through which certain aspects of the world (one's current situation, imagined future events, etc.) are experienced as emotionally salient (see also Colombetti 2014, chapter 5); because these bodily experiences are often subtle and inconspicuous, they can be mistaken for "purely mental" (Kriegel, 2015, p. 89). To illustrate this second possibility, consider again Armon-Jones's example of her experience of an alleged "non-bodily" envy. As she describes this case, she says that her thoughts "are totally consumed by a 'strong desire for an object which I do not possess and which belongs to another' ", and that these thoughts exhaust her experience of envy. But it does not seem to be phenomenologically accurate to say that one can be "totally consumed" by a "strong desire" (note the intensity of the 
described experience) for an object without any bodily phenomenology. Even if we grant that the desired object is quite abstract (e.g., respect and admiration, rather than a new modern kitchen) and that being "totally consumed" by a desire for it consists in cognitive rumination, it seems inaccurate to maintain that bodily feelings are entirely absent from this experience. In particular, we suggest that the experience of, e.g., envy, is shaped by inconspicuous background bodily feelings that "colour" what the person attends to in her thoughts and ruminations (much like looking through a coloured pair of lenses makes the world show up as coloured, while the lenses themselves are not noted, and are rather that through which the world appears tinted). These background bodily feelings may not be noted by the envious person, but they nevertheless contribute to feelings of tension and unpleasantness that, arguably, partly constitute the experience of envy described by Armon-Jones. In addition, emotional experiences typically take place in the context of more general mood experiences - such as feeling up or down, sluggish or energised, tense or calm (Thayer, 1996). These feelings, we propose, are bodily feelings that constitute a "background bodily phenomenology" against which the occurrent emotional experiences can stand out (in the foreground). ${ }^{9}$ So, even if one granted that, sometimes at least, emotional experiences include primarily "purely mental" cognitive or conative phenomenology, it would still be the case that bodily feelings constituting background moods shape and structure what is in the foreground. Taken together, these considerations complement, at the experiential level, the point we made in the previous section about the existence of several bidirectional pathways between brain and body, such that it does not seem possible to "silence" all sources of feedback from the body.

\section{Summary and conclusion}

In sum, then, in this chapter we have argued that the notion of arousal used in affective science needs to be characterised broadly, both at the physiological and experiential level, and cannot be reduced to narrow conceptions of interoception

9 See also Damasio (1999) for the related notion of "background feeling", and Colombetti (2014, chapter 5) for a discussion of this and other notions of background bodily experiences. 
limited to the perception (conscious and not) of ANS activation. At the physiological level, we need to move beyond Cannon's (1929) and Schachter \& Singer's (1962) conceptualisation of arousal as a uniform pattern of sympathetic activation. The sympathetic and parasympathetic systems are complexly interrelated and work together to generate specific patterns of autonomic arousal; additionally, autonomic activity includes not just neural efferent processes, but is also influenced by afferent neural and humoral feedback. Third, autonomic activation does not happen in isolation from other bodily processes, rather it is complexly interrelated via bidirectional pathways with the CNS, and the endocrine and immune system. Fourth, we suggested that the notion of physiological arousal may be extended even beyond processes taking place within the organism traditionally conceived, so as to include (at least) those gut bacteria known to influence, and be influenced by, neural, endocrine, and immune processes relevant to emotions and moods.

At the experiential level, we have argued that arousal cannot be reduced to visceral sensations of autonomic origin, but instead additionally includes perception of circulating substances mediated via humoral interoceptive pathways as well as specific somatic sensations: feedback from facial muscles and bodily posture, and felt urges to act (perception of muscle tension in preparation for action). We also proposed to regard "background bodily feelings" as constituting the experience of being aroused during emotion, where these are feelings in which bodily selfawareness is present but is not conspicuous, and is best characterised as shaping the person's experience from, or through, the background.

\section{References}

Aristotle. (1986). De Anima (On the Soul). (H. Lawson-Tancred, Trans.). London: Penguin.

Armon-Jones, C. (1986). The Thesis of Constructionism. In R. Harré (Ed.), The social construction of emotions (pp. 32-56). Oxford: Blackwell.

Ax, A. F. (1953). The Physiological Differentiation between Fear and Anger in Humans. Psychosomatic Medicine, 15(5), 433-442.

Barbur, J. L. (2004). Learning from the Pupil: Studies of Basic Mechanisms and Clinical Applications. In L. M. Chalupa \& J. S. Werner (Eds.), The visual 
neurosciences (Vol. 1, pp. 641-656). Cambridge MA: MIT Press.

Berntson, G. G., \& Cacioppo, J. T. (2009). Autonomic Nervous System. In D. Sander

\& K. R. Scherer (Eds.), The Oxford companion to emotion and the affective sciences (pp. 65-67). Oxford: Oxford University Press.

Bradley, M. M., \& Lang, P. J. (2007). Emotion and Motivation. In J. Cacioppo, L. G.

Tassinary, \& G. G. Berntson (Eds.), The handbook of psychophysiology (Third Edition, pp. 581-607). New York: Cambridge University Press.

Cannon, W. B. (1929). Bodily changes in pain, hunger, fear and rage (Second Edition). Boston: Charles Branford.

Charmandari, E., Tsigos, C., \& Chrousos, G. (2005). Endocrinology of the Stress Response. Annual Review of Physiology, 67(1), 259-284.

Colombetti, G. (2014). The feeling body: Affective science meets the enactive mind. Cambridge, MA: MIT Press.

Colombetti, G., \& Kuppens, P. (forthcoming). Valence, Arousal and their Relation: Conceptual Clarifications and Empirical Issues. In A. Scarantino (Ed.), The Routledge handbook of emotion theory. Oxon: Routledge.

Critchley, H. D., \& Harrison, N. A. (2013). Visceral Influences on Brain and Behavior. Neuron, 77(4), 624-638.

Cryan, J. F., \& Dinan, T. G. (2012). Mind-Altering Microorganisms: The Impact of the Gut Microbiota on Brain and Behaviour. Nature Reviews Neuroscience, 13(10), 701-712.

Damasio, A. R. (1999). The feeling of what happens: Body, emotion and the making of consciousness. London: Vintage.

Dantzer, R., O'Connor, J. C., Freund, G. G., Johnson, R. W., \& Kelley, K. W. (2008). From Inflammation to Sickness and Depression: When the Immune System Subjugates the Brain. Nature Reviews Neuroscience, 9(1), 46-56.

Davidson, R.J., Scherer, K.R., \& Goldsmith, H.H. (Eds.)(2003). Handbook of affective aciences. Oxford: Oxford University Press.

Dixon, T. (2003). From passions to emotions: The creation of a secular psychological category. Cambridge: Cambridge University Press.

Dror, O. E. (2014). The Cannon-Bard Thalamic Theory of Emotions: A Brief Genealogy and Reappraisal. Emotion Review, 6(1), 13-20.

Foster, J. A., \& McVey Neufeld, K.-A. (2013). Gut-Brain Axis: How the Microbiome Influences Anxiety and Depression. Trends in Neurosciences, 36(5), 305- 
312.

Fowles, D. C. (2009). Arousal. In D. Sander \& K. R. Scherer (Eds.), The Oxford companion to emotion and the affective sciences (p. 50). Oxford: Oxford University Press.

Fox, E. (2008). Emotion science: Cognitive and neuroscientific approaches to understanding human emotions. Basingstoke: Palgrave Macmillan.

Frijda, N. H. (1986). The emotions. Cambridge: Cambridge University Press.

Garfinkel, S. N., Seth, A. K., Barrett, A. B., Suzuki, K., \& Critchley, H. D. (2015). Knowing Your Own Heart: Distinguishing Interoceptive Accuracy from Interoceptive Awareness. Biological Psychology, 104, 65-74.

Gill, C. (2010). Naturalistic psychology in Galen and Stoicism. Oxford, New York: Oxford University Press.

Graver, M. R. (2007). Stoicism and emotion. Chicago: University Of Chicago Press. Grewe, O., Nagel, F., Kopiez, R., \& Altenmuller, E. (2007). Emotions Over Time: Synchronicity and Development of Subjective, Physiological, and Facial Affective Reactions to Music. Emotion, 7, 774-788.

Harrison, N. A. (2017). Brain Structures Implicated in Inflammation-Associated Depression. Current Topics in Behavioral Neuroscience, 31, 221-248.

Harrison, N. A., Kreibig, S. D., \& Critchley, H. D. (2013). A Two-Way Road: Efferent and Afferent Pathways of Autonomic Activity in Emotion. In J. Armony \& P. Vuilleumier (Eds.), The Cambridge handbook of human affective neuroscience (pp. 82-106). Cambridge: Cambridge University Press.

Hume, D. (1739-40 [2003]). A treatise of human nature. London: Everyman. James, W. (1884). What is an Emotion? Mind, 9, 188-205.

Jänig, W. (2006). The integrative action of the autonomic nervous system:

Neurobiology of homeostasis. Cambridge: Cambridge University Press.

Kragel, P. A., \& LaBar, K. S. (2016). Decoding the Nature of Emotion in the Brain. Trends in Cognitive Sciences, 20(6), 444-455.

Kriegel, U. (2015). The varieties of consciousness. Oxford: Oxford University Press. Langley, J. N. (1900). The Sympathetic and Other Related Systems of Nerves. In E. A. Sharpey-Schäfer (Ed.), Textbook of physiology (pp. 616-696). London: Young J. Pentland.

Mayer, E. A., Knight, R., Mazmanian, S. K., Cryan, J. F., \& Tillisch, K. (2014). Gut Microbes and the Brain: Paradigm Shift in Neuroscience. The Journal of 
Neuroscience, 34(46), 15490-15496.

Nummenmaa, L., Glerean, E., Hari, R., \& Hietanen, J. K. (2014). Bodily Maps of Emotions. Proceedings of the National Academy of Sciences, 111(2), 646651.

Rimé, B., Philippot, P., \& Cisamolo, D. (1990). Social Schemata of Peripheral Changes in Emotion. Journal of Personality and Social Psychology, 59(1), 38-49.

Rivest, S. (2009). Regulation of Innate Immune Responses in the Brain. Nature Reviews. Immunology, 9(6), 429-439.

Robinson, B. F., Epstein, S. E., Beiser, G. D., \& Braunwald, E. (1966). Control of Heart Rate by the Autonomic Nervous System: Studies in Man on the Interrelation between Baroreceptor Mechanisms and Exercise. Circulation Research, 19(2), 400-411.

Russell, J. A. (2003). Core Affect and the Psychological Construction of Emotion. Psychological Review, 110(1), 145-172.

Saper, C. B., Romanovsky, A. A., \& Scammell, T. E. (2012). Neural Circuitry Engaged by Prostaglandins During the Sickness Syndrome. Nature Neuroscience, 15(8), 1088-1095.

Scarantino, A. (Ed.)(forthcoming). The Routledge handbook of emotion theory. Oxon: Routledge.

Schachter, S. (1964). The Interaction of Cognitive and Physiological Determinants of Emotional State. In L. Berkowitz (Ed.), Advances in experimental social psychology (pp. 49-80). New York: Academic Press.

Schachter, S., \& Singer, J. (1962). Cognitive, Social, and Physiological Determinants of Emotional State. Psychological Review, 69(5), 379-399.

Scherer, K. R., \& Wallbott, H. G. (1994). Evidence for Universality and Cultural Variation of Differential Emotion Response Patterning. Journal of Personality and Social Psychology, 66, 310-328.

Schulz, A., Lass-Hennemann, J., Sütterlin, S., Schächinger, H., \& Vögele, C. (2013). Cold Pressor Stress Induces Opposite Effects on Cardioceptive Accuracy Dependent on Assessment Paradigm. Biological Psychology, 93(1), 167-174.

Spiga, F., Walker, J. J., Gupta, R., Terry, J. R., \& Lightman, S. L. (2015). 60 Years of Neuroendocrinology. Glucocorticoid Dynamics: Insights from Mathematical, Experimental and Clinical Studies. Journal of Endocrinology, 226(2), T55- 
T66.

Sudo, N., Chida, Y., Aiba, Y., Sonoda, J., Oyama, N., Yu, X.-N., .. Koga, Y. (2004). Postnatal Microbial Colonization Programs the Hypothalamic-PituitaryAdrenal System for Stress Response in Mice. The Journal of Physiology, 558(1), 263-275.

Thayer, R. E. (1996). The origin of everyday moods: Managing energy, tension, and stress. Oxford: Oxford University Press.

Tracey, K. J. (2002). The Inflammatory Reflex. Nature, 420(6917), 853-859.

Wiens, S., Mezzacappa, E. S., \& Katkin, E. S. (2000). Heartbeat Detection and the Experience of Emotions. Cognition \& Emotion, 14(3), 417-427.

Wolf, S., \& Wolff, H. G. (1947). Human gastric function. New York: Oxford University Press. 